\title{
Electron-positron outflow from black holes and the formation of winds
}

\author{
Maurice H.P.M. van Putten \\ MIT, Cambridge, MA 02139
}

\begin{abstract}
The collapse of young massive stars or the coalescence of a black holeneutron star binary is expected to give rise to a black hole-torus system. When the torus is strongly magnetized, the black hole produces electron-positron outflow along open magnetic field-lines. Through curvature radiation in gaps, this outflow rapidly develops into a $e^{ \pm} \gamma$-wind, which is ultra-relativistic and of low comoving density, proposed here as a possible input to GRB fireball models.
\end{abstract}

Here, I discuss some aspects of black holes when exposed to external magnetic fields. For example, black hole-torus systems are a probable outcome of the collapse of young massive stars $[28,17]$ and the coalescence of black hole-neutron star binaries [18], both of which are possible progenitors of cosmological gamma-ray bursts (GRBs). If all black holes are produced by stellar collapse, they should be nearly maximally rotating $[1,2]$. A surrounding torus or accretion disk is expected to be magnetized by conservation of magnetic flux and linear amplification (cf. $[17,15])$.

A black hole-torus system will have open magnetic field-lines from the horizon to infinity and closed magnetic field-lines between the black hole and the torus [23]. The closed magnetic field-lines mediate Maxwell stresses [24]. This may be seen by way of similarity to pulsar magnetospheres [10]. In a poloidal crosssection, the torus can be identified with a pulsar which rotates at an angular velocity $\Omega_{P} \sim \Omega_{H}-\Omega_{T}$, wherein the black hole horizon corresponds to infinity. Then, the inner light-surface [29] corresponds to the pulsar light-cylinder, and a 'bag' attached to the torus to the last closed field-line. Here, $\Omega_{H}$ and $\Omega_{T}$ denote the angular velocities of the black hole and the torus, respectively. The work performed by the Maxwell stresses is commonly attributed to an outgoing Poynting flux emanating from the horizon [3,21]. These Maxwell stresses are likely to be important to the evolution of the torus, and tend to delay accretion onto the black hole. The open magnetic field-lines, on the other hand, enable the black hole to produce an outflow to infinity. Such outflows generate emissions by deceleration against the interstellar medium and through internal shocks. 
Here, the outflow along open magnetic field-lines is studied, and found to produce a pair-dominated $e^{ \pm} \gamma$-wind in combination with curvature radiation.

Open field-lines from the horizon to infinity have radiative ingoing boundary conditions at the horizon as seen by zero-angular momentum observers (ZAMOs), and outgoing boundary conditions at infinity. It is well-known that for an outflow to exist, there must be regions in which pairs are created (gaps), somewhere on these open field-lines $[3,19,20,4]$. The gaps are powered by an electric current $I$ along the field-lines, which is limited by a horizon surface resistivity of $4 \pi$, in the presence of a certain potential drop across them. The net particle flow is limited by the black hole luminosity into the gap. The magnetosphere within the gaps is differentially rotating, beyond which the magnetosphere may be force-free and in rigid rotation. Note that, in contrast, the currents along closed magnetic fieldlines are fixed by the angular velocity $\Omega_{T}$ of the surrounding matter, where the gaps are most likely residing between the horizon and the inner light surface. Of interest here is the location of the gaps on the open magnetic field-lines and the power dissipated within, as sites of linear acceleration of charged particles and their curvature radiation.

A rotating black hole tends to produce electrons and positrons by spontaneous emission along open magnetic field-lines in an effort to evolve to a lower energy state by shedding off its angular momentum. Indeed, in the adiabatic limit, the radiated particles possess a specific angular momentum of at least $2 M$, whereas the specific angular momentum of the black hole, $a$, is at most $M$. In the approximation of an asymptotically uniform magnetic field, e.g., in a Wald-field [27], the emissions at infinity to satisfy a Fermi-Dirac distribution of radiative Landau states, neglecting curvature radiation and magnetic mirror effects. This results from a modification to the Hawking radiation process [25]. This is a highly idealized picture derived in the perturbative limit of small particle densities, which will be modified significantly by curvature radiation and the formation of force-free regions. The spontaneous emission process concerns particles with energy-at-infinity $\omega$ below the Fermi-level $V_{F}$. Here, $V_{F}$ is the energy-at-infinity associated with the particles as seen on a null-generator of the horizon, such as the ZAMO-derivative $\xi^{a} \partial_{a}=\partial_{t}-\beta \partial_{\phi}$, where $\beta$ denotes the angular velocity of the sky as seen by ZAMOs. That is,

$$
V_{F} \psi=\left[\xi^{a} D_{a}\right]_{\infty}^{H} \psi=\left(\nu \Omega_{H}-e V\right) \psi
$$

where $\Omega_{H}$ is the angular velocity of the horizon, using the sign-convention $\psi \propto$ $e^{-i \omega t} e^{i \nu \phi}$. The energy-at-infinity $\omega$ and the azimuthal quantum number $\nu$ are associated with the asymptotically time-like Killing vector $\partial_{t}$ and azimuthal Killing vector $\partial_{\phi}$, whereas $D_{a}=i^{-1} \partial_{a}+e A_{a}$ denotes the gauge-covariant derivative in the presence of an electromagnetic vector potential $A_{a}$. In calculating $V_{F}$, it is relevant to identify the ground state of the black hole-magnetic field configuration. It has been shown that the lowest energy state, in the process of an angular momentum exchange between the black hole and the surrounding electromagnetic field by variations of the horizon charge $q$, assumes when $q=2 B J[27,7]$, where $J$ is the 
angular momentum of the black hole. Rotation of the equilibrium charge $q=2 B J$ on the horizon recovers $4 \pi B M^{2}$ as the maximal horizon flux of the magnetic field from the uncharged flux $4 \pi B M^{2} \cos \lambda$, where $\sin \lambda=J / M^{2}[7,25]$. With the sign convention that $B$ is parallel to $\Omega_{H}$, we then have $V_{F}=\nu \Omega_{H}$ with $\nu=e A_{\phi}$ (for $\left.e^{-}\right)$and $A_{a}=B\left(\partial_{\phi}\right)_{a} / 2$ in the Wald electrostatic equilibrium state. Note that the spontaneous emission process is anti-symmetric under pair-conjugation.

The rate of spontaneous emission is given by a certain barrier transmission coefficient in the level-crossing picture of electrons and positrons [6]. This follows from frame-dragging by $\beta$, and the resulting shift between the energy-at-infinity $\omega$ and the energy $\omega_{Z}$ as seen by ZAMOs:

$$
\omega_{Z}= \pm \sqrt{m_{e}^{2}+|e B|(2 n+1 \pm \alpha)}=\omega+\nu \beta=\left\{\begin{array}{cc}
\omega-\nu \Omega_{H} & \text { on the horizon } \\
\omega & \text { at infinity. }
\end{array}\right.
$$

Here, it is the quantum number $\nu$ which gives rise to different energies between ZAMOs and Boyer-Linquist observers. Figure 1 (a) shows an equivalent classical picture, where the frame-dragging $\beta$ induces a potential energy $V_{B L}=e \beta A_{\phi}$ on a flux surface $A_{\phi}=$ const. with respect to the axis of rotation, itself at zero potential in the $q=2 B J$ state. Since $\beta$ describes a differentially rotating space-time, it varies with distance to the black hole and $V_{B L}$ introduces a potential energy drop along the magnetic field-lines. When sufficiently strong, a Schwinger-type process is set in place, which locally produces pairs at a certain rate per unit volume. Formally, the rate of pair-production follows from a scattering calculation in the WKB approximation [14,9,6] (cf. also [13]). The pair-production rate is found to be given by a barrier transmission coefficient $\Gamma \sim e^{-\pi B_{c} / B \theta^{2}}$, where $B_{c}=m_{e}^{2} / e=4.4 \times$ $10^{13} \mathrm{G}$ is the QED value of the magnetic field-strength and $\theta$ is the poloidal angle in Boyer-Linquist coordinates. More precisely, the gradient $\eta=-\nabla V_{B L}$ parallel to $B$ drives a pair-production rate per unit volume by a Schwinger-process $d^{2} N / d t d V \sim$ $\left(e^{2} \eta B / 4 \pi^{2}\right) e^{-\pi B_{c} / \eta \theta^{2}} \quad\left(B>>B_{c}, a \sim M\right)$. This pair-production process will be in place, whenever the charge-density is low so that the magnetosphere remains in differential rotation.

The magnetosphere on open field-lines away from a gap assumes a force-free, rigidly rotating state with a Goldreich-Julian charge density [3,21,22]. This is similar to the analogous case in pulsar magnetospheres [11,12]. In view of the horizon boundary conditions below, I shall assume that the gap is attached to the horizon.

To a first approximation, the local structure of a gap follows from the ingoing radiative horizon-boundary conditions. The flow in a gap is described by a chargedensity $\rho_{e}$, a pair-density $n_{w}$ and a Lorentz factor $\Gamma$. This flow is powered by an electric current $I$ along the open field-lines to infinity through a polar cap of area $A_{p}$ at the cost of a certain potential drop across. The ingoing radiative boundary condition at the horizon applies to electrons and positrons alike: in the limit as we approach the horizon, $I$ and the electric charge density $\rho_{e}$ are no longer independent, but become proportional to one another (cf. [20]): 


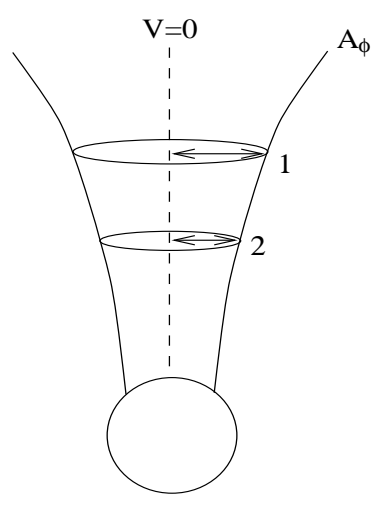

(a)

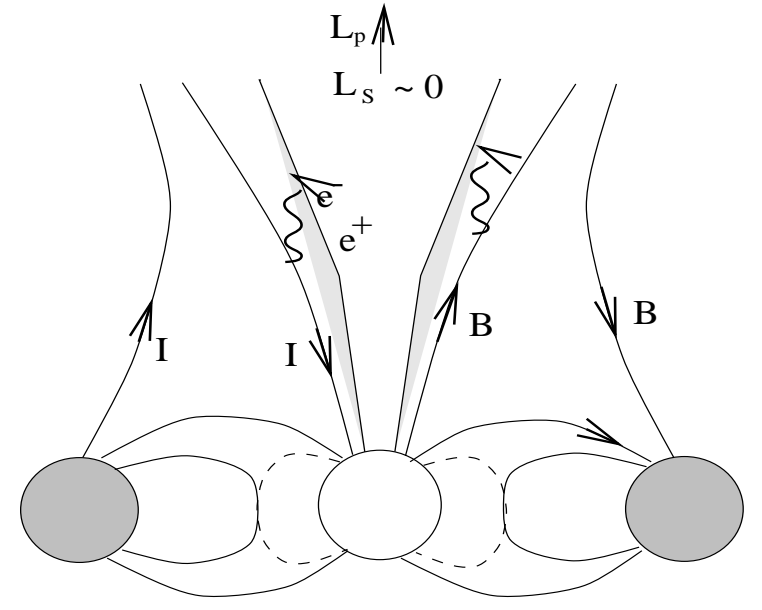

(b)

Figure 1. (a) A classical picture of the potential energy $V_{B L}$ as seen in Boyer-Lindquist coordinates along surfaces of constant magnetic flux $A_{\phi}$. Note that the axis of rotation has zero potental $V=0$ in electrostatic equilibrium $q=2 B J$. Hence, $V_{B L}=e \beta A_{\phi}$ in the presence of frame-dragging $\beta$. Since $\beta$ describes differential rotation in the surrounding space-time, a potential drop emerges along $A_{\phi}=$ const.: $\Delta V_{B L}=\left(\beta_{2}-\beta_{1}\right) A_{\phi}$. When the potential drop is steep, a Schwinger-type process generates electron-positron pairs. (b) Cartoon of the formation of a black hole-wind in a black hole-torus system. There is a minimum opening angle $\theta_{\text {min }} \sim \sqrt{B_{c} / 3 B}$, beyond which spontaneous emission along open field-lines by the black hole is effective. Flux surfaces with $\theta \sim \theta_{\min }$ have a gap length of order $M$, which decreases for $\theta>\theta_{\min }$. These gaps, indicated in grey, create pairs, which are subject to linear acceleration and produce curvature radiation. The net outflow $L_{p}$ in particles is a combination of an inner, current-free outflow with vanishingly small Poynting flux $L_{S}$ inside $\theta<\theta_{\text {min }}$ and an outer, current-carrying outflow with $\theta>\theta_{\text {min }}$. Both derive most of their particles from pair-cascade through curvature radiation, and flow along open field-lines to infinity. 


$$
I \longrightarrow-\rho_{e} A_{p},
$$

since all particles fall into the black hole with the velocity of light. The sign of $\rho_{e}$ in (3) is that seen by ZAMOs. Here, $\rho_{e}$ (and $n_{w}$ ) are normalized by factoring in the redshift factor. $I$ saturates against the horizon surface resistivity of $4 \pi: 4 \pi I \sim \nu \Omega_{H}$, up to a logarithmic factor on the left hand-side. Hence, $\rho_{e} \sim \rho_{G J} / 2$, where $\rho_{G J}=$ $B \Omega_{H} / 2 \pi$ is the Goldreich-Julian charge density near the horizon. With curvature $R_{B} \sim \sqrt{2} M / \theta^{2}$ of the Wald-field, curvature radiation produces $n_{w}>>\rho_{e} / e$ in momentum balance: $n_{w} 2 e^{2} \Gamma^{4} / 3 R_{B}^{2} \sim \rho_{e} E_{\perp}$, where $E_{\perp} \sim \nu \Omega_{H} / M e$ is the equivalent electric field normal to the horizon as seen in Boyer-Lindquist coordinates. Note, however, that the magnetic field of the torus will have larger curvature than that of the Wald-field. Given energy balance of the outflow $n_{w} \Gamma m_{e} A_{c a p}$ with the total power $I E_{\perp} L$ dissipated in the gap, where $L$ is the linear gap size, the solutions are governed by the unknown $L$.

The gap size $L$ determines the degree to which the black hole luminosity is put to work in accelerating particles. The gap produces a radiation pressure $\propto L$, which acts on the interface with the force-free magnetosphere above. The interface is probably Raleigh-Taylor unstable against this radiation pressure. Moreover, the gap itself may well widen due to this pressure. The arguments given above are intended as a first sketch towards the structure of the gaps, and it appears to be of interest to consider the gap size $L$ in the context of a detailed stability analysis.

A continuous outflow establishes with appropriate current closure. Note that closure over the polar axis introduces Poynting flux with negative helicity, whereas current closure over a gap across the equator of the black hole and the bag of the torus (corresponding to the last field-line in pulsar magnetospheres) introduces Poynting with positive helicity - indicative of positive energy and angular momentum transport outwards. As the latter is energetically favorable over the former, thereby leaving negligible Poynting flux over the axis of symmetry. A similar conclusion has been found in the case of current closure around neutron stars [11,12]. It follows that the black hole-wind is pair-dominated with the property that $\sigma=L_{S} / L_{p} \sim 0$

within $\theta<\theta_{\min }=\sqrt{B_{c} / 3 B}$, where $L_{S}$ and $L_{p}$ are the luminosities in Poynting flux and pairs, respectively [26]. Figure 1 (b) sketches this wind-formation process, assuming a $L$ to be large on the flux surfaces with $\theta \sim \theta_{\text {min }}$.

It will be of interest to look for observational evidence in GRB-afterglow emissions for the presented ultra-relativistic, low density pair-dominated wind.

\section{Acknowledgements}

This work is partially supported by NASA Grant 5-7012 and an MIT Reed Award. The author thanks the hospitality of Theoretical Astrophysics, Caltech, and the Korean Institute of Advanced Study (KIAS), where some of this work was performed, and gratefully acknowledges stimulating discussions with P. Goldreich, E.S. Phinney, R.D. Blandford and K.S. Thorne. 


\section{REFERENCES}

1. Bardeen J.M., Nature, 226, 64 (1970).

2. Bethe H.A. \& Brown G.E., astro-ph/9805355.

3. Blandford R.D. \& Znajek R.L. Mon. Not. R. Astron. Soc., 179:433-456 (1977).

4. Beskin V.S. \& Kuznetsova I.V., Il Nuovo Cimento (to appear, 2000).

5. Chevalier R.A. and Li Z.-Y., astro-ph/9908272 (1999).

6. Damour T. in Proc. $1^{\text {st }}$ Marcel Grossmann Meeting on Gen. Rel., ed.R. Ruffini (North Holland, Amsterdam, 1977), p459-482.

7. Dokuchaev V.I. Sov. Phys. JETP, 65(6):1079-1086 (1987).

8. Gavrilov S.P. \& Gitman D.M., Phys. Rev. D, 53:7162 (1996).

9. Gibbons G.W. MNRAS, 177:37P (1976).

10. Goldreich P. \& Julian W.H., ApJ., 157:869 (1969).

11. Goldreich P., in Publ Astron Soc Pacific, 83(495):599 (1971).

12. Goldreich P., in Accademia Nazionale Dei Lincei, N. 162, p151 (1971).

13. Goldreich P. \& Tremaine S., ApJ., 222:850 (1978).

14. Hawking S.W. Commun. Math. Phys., 43:199 (1975).

15. Kluzniak W. \& Ruderman M., ApJ., 505:L113 (1998).

16. Lin Q., hep-th/9810037 (1998).

17. Paczyński B. ApJ., 494:45 (1998).

18. Paczyński B. Acta Astron., 41: 257-267 (1991).

19. Phinney E.S., in Astrophysical Jets (Reidel, Dordrecht 1983), p201.

20. Punsly B. \& Coroniti F.V., Phys. Rev. D., 40:3834 (1989); ibid. ApJ., 350:518 (1990).

21. Thorne K.S., Price R.H. and Macdonald D.A. Black holes: the membrane paradigm (Yale Univ. Press, 1986).

22. Treves A., Turolla R. \& Popov S.B., astro-ph/0005508 (2000).

23. van Putten M.H.P.M. and Wilson A. in ITP Conference on black holes: Theory confronts reality, http://www.itp.ucsb.edu/online/bhole-c99 (1999).

24. van Putten M.H.P.M. Science, 284:115-118 (1999).

25. van Putten M.H.P.M., Phys. Rev. Lett., 84(17):3752 (2000).

26. van Putten M.H.P.M., http://online.itp.ucsb.edu/online/astro99.

27. Wald R.M., Phys. Rev. D., 10:1680-1684 (1974).

28. Woosley S., ApJ., 405:273-277 (1993).

29. Znajek R.L., Mon. Not. R. Astron. Soc., 179:457-472 (1977). 